\title{
Liner Shipping Hub Network Design in a Competitive Environment
}

\author{
Gelareh, Shahin; Nickel, Stefan; Pisinger, David
}

Publication date:

2010

Document Version

Publisher's PDF, also known as Version of record

Link back to DTU Orbit

Citation (APA):

Gelareh, S., Nickel, S., \& Pisinger, D. (2010). Liner Shipping Hub Network Design in a Competitive Environment. DTU Management. DTU Management 2010 No. 6

http://www.man.dtu.dk/upload/institutter/ipl/publ/publikationer\%202010/rapport\%206.1.pdf

\section{General rights}

Copyright and moral rights for the publications made accessible in the public portal are retained by the authors and/or other copyright owners and it is a condition of accessing publications that users recognise and abide by the legal requirements associated with these rights.

- Users may download and print one copy of any publication from the public portal for the purpose of private study or research.

- You may not further distribute the material or use it for any profit-making activity or commercial gain

- You may freely distribute the URL identifying the publication in the public portal

If you believe that this document breaches copyright please contact us providing details, and we will remove access to the work immediately and investigate your claim 
Liner Shipping Hub Network Design in a Competitive Environment

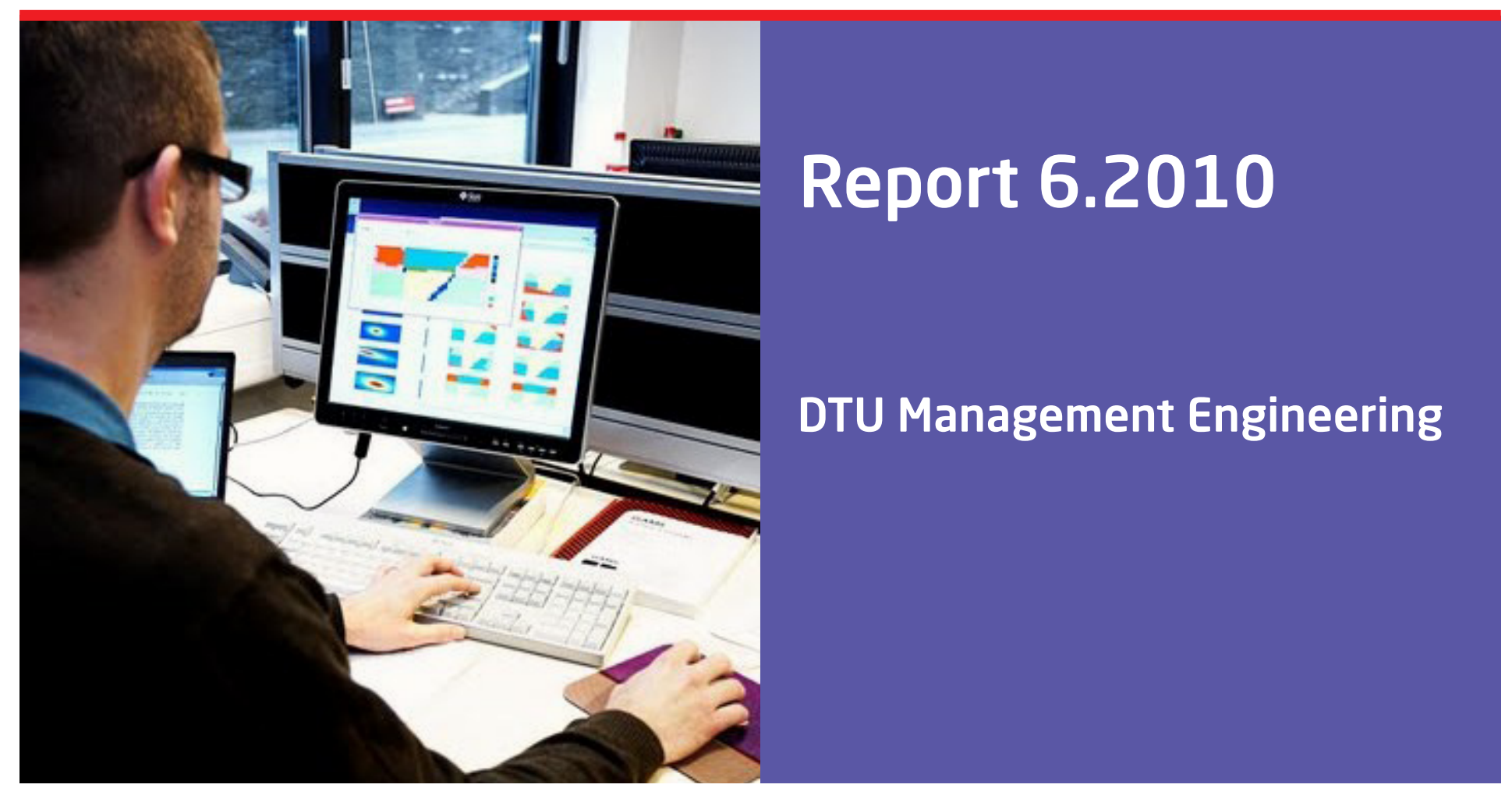

Shahin Gelareh Stefan Nickel

David Pisinger

February 2010 


\title{
Liner Shipping Hub Network Design in a Competitive Environment
}

\author{
Shahin Gelareh ${ }^{\mathrm{a}, 1, *}$, Stefan Nickel $^{\mathrm{b}}$, David Pisinger ${ }^{\mathrm{a}}$ \\ ${ }^{a}$ Department of Management Engineering, Technical University of Denmark, Produktionstorvet, \\ Building 426, 2800 Kgs. Lyngby, Denmark \\ ${ }^{b}$ Institut für Operations Research, Universität Karlsruhe, D-76128 Karlsruhe, Germany
}

\begin{abstract}
A mixed integer programming formulation is proposed for hub-and-spoke network design in a competitive environment. It addresses the competition between a newcomer liner service provider and an existing dominating operator, both operating on hub-and-spoke networks. The newcomer company maximizes its market share-which depends on the service time and transportation cost—by locating a predefine number of hubs at candidate ports and designing its network. While general-purpose solvers do not solve instances of even small size, an accelerated Lagrangian method combined with a primal heuristic obtains promising bounds. Our computational experiments on real instances of practical size indicate superiority of our approach.
\end{abstract}

Keywords: hub-and-spoke network design, liner shipping, competition, mixed integer programming, Lagrangian relaxation, enumeration.

\section{Introduction}

During the last two decades, hub-and-spoke network design problems have received increasing attention in a wide range of application areas such as transportation, telecommunications, computer networks, postal delivery, lessthan-truck loading (LTL) and supply chain management. The economy of scale offered by hub-and-spoke network structures for transferring origin-destination (O-D) $\mathrm{fl}$ ws is exploited by concentrating $\mathrm{fl} \mathrm{w}$ on fewer links and by avoiding underutilized connections.

Aiming at minimizing the total costs in a hub-and-spoke network, $\mathrm{fl}$ w between O-D pairs is routed through some selected intermediate nodes (called hub nodes) and edges (called hub edges) connecting the hubs. Once the hubs are chosen, the non-hub nodes (called spoke nodes) are allocated to them in order to transship fl w via the hub-level sub-network. The allocation scheme is either single or multiple based on the particular nature of application. In a single allocation scheme, a spoke node is allocated to a single hub, while such restriction is relaxed in a multiple allocation scheme. Such a hub-and-spoke structure avoids direct shipping, which result in underutilized (or at least very infrequently utilized) use of vessels operating on some (or perhaps many) of the direct links and drops the underutilized links in favor of concentrating $\mathrm{fl} \mathrm{w}$ on hub edges and better utilization of facilities operating there. As a result of this fl w concentration, economy of scale can be exploited by using more efficient vessels on the hub links.

Most of the Hub Location Problems (HLP) presented in the literature are based on four main assumptions (Nickel et al., 2001):

a. The hub-level network is a complete graph.

b. Using inter-hub connections has a lower price per unit than using spoke connections. The economy of scale is expressed by a discount factor $\alpha,(0<\alpha<1)$ on the inter-hub connections.

c. Direct connections between the spoke nodes are not allowed.

\footnotetext{
${ }^{*}$ Corresponding author (Tel: +4545253386$)$

Email address: shge@man.dtu.dk; shahin.gelareh@gmail.com (Shahin Gelareh)

${ }^{1}$ This work has been partially carried out during firs author's appointment at the Department of Civil Engineering, National University of Singapore
} 
d. Costs are proportional to the distance (and hence the triangle inequality holds).

Although classical models of HLPs have been introduced based on these assumptions, some of them might not be realistic in practice. Examples of such cases are transport services wherein the hub-level may not necessarily be a complete sub-graph — even completeness may not be physically feasible due to existing environmental barriers - and Euclidean metrics usually do not hold in the cost structure since freight costs are based on demand and competition. Moreover, there might be direct connections between spoke nodes if, for instance, the travel time needs to satisfy some given target.

\subsection{Literature review}

HLP Problems originate from Goldman's idea (Goldman, 1969) on node optimality. In his work, he proved that Hakimi's argument (Hakimi, 1964) holds even for more general cases and proposed two formulations. O'Kelly $(1986 a, b)$ was the firs to propose studying hub location problems. These papers deal with one and two hub systems in the plane. However, in the present paper, we deal with the discrete hub location problem. The firs work in this area is again due to O'Kelly (1987), where he proposed the firs (quadratic) mathematical formulation for the Uncapacitated Single Allocation p-Hub Median Problem (USA pHMP).

Since then many papers have studied various variants (single and multiple allocation, capacitated and uncapacitated, competitive, budget constraints etc.) of HLPs from different perspectives (formulation, polyhedral properties, solution approaches) as well as for different applications (in particular, transportation and telecommunications). In the following, we refer to some of the applications in transportation networks.

Freight Transport. Powell and Sheffi (1983) deal with the load planning problem of less-than-truckload motor carriers through determination of freight routes on the network to minimize costs while maintaining service. Hall (1989) examines the impact of overnight restrictions and time zones on the configuratio of an air freight network and location of hub terminal. Iyer and Ratliff (1990) find the location of accumulation centers in a guaranteed time distribution system. O'Kelly and Miller (1991) considered a single facility minimax hub location problem for siting a hub facility in order to minimize the most costly interaction between a set of fi ed nodes. Campbell (2005) takes into account the strategic network design for motor carriers in a hub-and-spoke framework. Racunica and Wynter (2005) formulated a nonlinear concave-cost hub location problem to identify the optimal location of intermodal freight hubs. Jeong et al. (2007) applies the hub-and-spoke network problem for railroad freight to fin transport routes, frequency of service, length of trains to be used, and transportation volume etc. Cunha and Silva (2007) models a hub-and-spoke network design for LTL trucking company in Brazil. Recent papers include Calık et al. (2008) for a hub covering problem over incomplete hub networks, Yaman (2009) for a hierarchical hub median problem with single assignment, Campbell (2009) for time definit transportation services and Alumur et al. (2009) for a single allocation incomplete hub networks for LTL trucking in Turkey.

Public Transport. Mathematical models and solution approaches are presented in Gelareh and Nickel (2007) and Nickel et al. (2001). Gelareh (2008) proposed several variants of hub location problems with a variety of hub-level configuration in particular addressing the public transport planning (see Gelareh and Nickel (2008a)). They also proposed the firs multi-period hub location problem for the same application (see Gelareh and Nickel (2008b)). Exact and heuristic solution methods for both single and multi-period models were developed, and computational results indicated that both are very efficient.

Air Transport. Jaillet et al. (1996) introduce fl w-based models for designing capacitated networks and routing policies suggesting the presence of hubs whenever they are cost efficient. The network design problem is concerned with the operation of a single airline with a f xed share of the market. Kuby and Gray (1993) considered a hub network design problem with stopovers and feeders for Federal Express. A recent work is due to Eiselt and Marianov (2008) where he deals with a competitive hub location problem where customers have gravity-like utility functions.

Maritime Transport. Aversa et al. (2005) proposed a mixed integer programming (MIP) model for locating a hub port in the East coast of South America. In 2008, Takano and Arai (2008) applied the quadratic model of O'Kelly and developed a genetic algorithm to solve instances of the problem. As opposed to many existing tight linear formulations, this work applies a nonlinear formulation; it neither allows more than one hub edge being used along any O-D path nor 
any spoke-spoke connection. Imai et al. (2009) presented a model for simultaneous hub-and-spoke network design and flee deployment problems in liner shipping. Their emphasis is on the empty container repositioning and their model hardly resembles a standard hub-and-spoke model. Imai et al. (2006) studies the viability of deploying megavessels by employing a non-zero sum two-person game with hub-and-spoke networks strategy for mega-vessels and multi-port calling for conventional ship size. Other results can be found in Hsu and Hsieh (2007) for a two-objective model to determine the optimal liner routing, ship size, and sailing frequency for container carriers by minimizing shipping costs and inventory costs. Konings (2006) tries to answer the question whether hub-and-spoke services could be a fruitful tool in improving the performance of Container-on-barge transport and so in gaining market share. Other works addressing liner shipping network design, but not necessarily the hub-and-spoke design, include Choong et al. (2002) for empty container management for intermodal transportation networks. For a recent survey of models, applications and solution methods for network hub location problems, we refer to the survey by Alumur and Kara (2008) and the references therein.

Despite the large number of papers on hub location problems, two aspects have received much less attention in the literature: Competition and applications in maritime transportation.

To the best of our knowledge, Marianov et al. (1999) was the firs to formulate the competitive hub location problem arising in passenger and cargo transportation wherein either the increased passenger fl $\mathrm{w}$ or resulting revenue is maximized. In this study, they maximized the market share of a company (measured in the number of customers) by locating new hubs and motivating customers to change handler by reducing the fares. They assumed one or at most two hub nodes are visited along any O-D path and proposed a Tabu search for solving the problem.

Sasaki et al. (1999) formulated a continuous hub location model. In their model, two companies, one as a leader and the other one as a follower, are competing to locate their facilities. The problem is formulated as a bi-level programming model. Results show that the leader company share may be highly threatened by the competitor, if its decisions are not conformed with the decision of the competitor.

Sasaki and Fukushima (2001) proposed the so-called Stackelberg Hub Location Problem (SHLP) as an extension to Sasaki et al. (1999) where a big company is competing with several medium size companies and the game takes place between the leader company and the set of medium size companies. The medium size companies are mutually disjoint and not competing with each other. The leader's problem is again formulated as a bi-level programming problem. The bigger company decides firs to locate its hub in a continuous space and followers make the optimal decision based on that. The model is solved using sequential quadratic programming methods. Again, it is assumed that only one or two intermediate hubs are used on each path.

Wagner (2008) suggested some modification to the models presented in (Marianov et al., 1999), and noted that the proposed Tabu search method contains a faw. However it is still assumed that the cost structure satisfie the triangle inequality.

Eiselt and Marianov (2008) extended the work by Marianov et al. (1999) — in which the market share was only a function of the cheapest rates - to a nonlinear model aiming at maximizing the share by means of a utility function. Their utility function is a function of time, cost and attractiveness. Such an attractiveness function was firs proposed by Drezner and Drezner (2001).

\subsection{Objective and Contribution}

When compared to most other modes of transportation, liner shipping offers cheaper freight rates, higher safety level and less environmental impact. However, compared to other services, the liner transportation problem has received less attention in the literature. The current trend of global trade and emerging new economies UNCTAD (2008) - in particular in Asia - enhance the importance of liner shipping, since it is in charge of transporting up to 90 percent of this trade volume. To avoid deployment of underutilized vessels across transpacifi and transatlantic trade routes a number of alliances have recently emerged. Several transshipment hubs are being identifie and companies are collaborating within alliances to achieve higher economy of scale by deploying proper container-ship sizes across the transportation network.

This had led to a situation where several of the alliances have a dominating position, and most of the smaller liner service providers (LSP) are suffering from an ongoing loss of their market shares. It is therefore essential for the smaller liner service providers - that are not operating in alliances - to seek for ways of competing with the alliances in order to achieve some higher market shares or at least to recover the lost shares. 


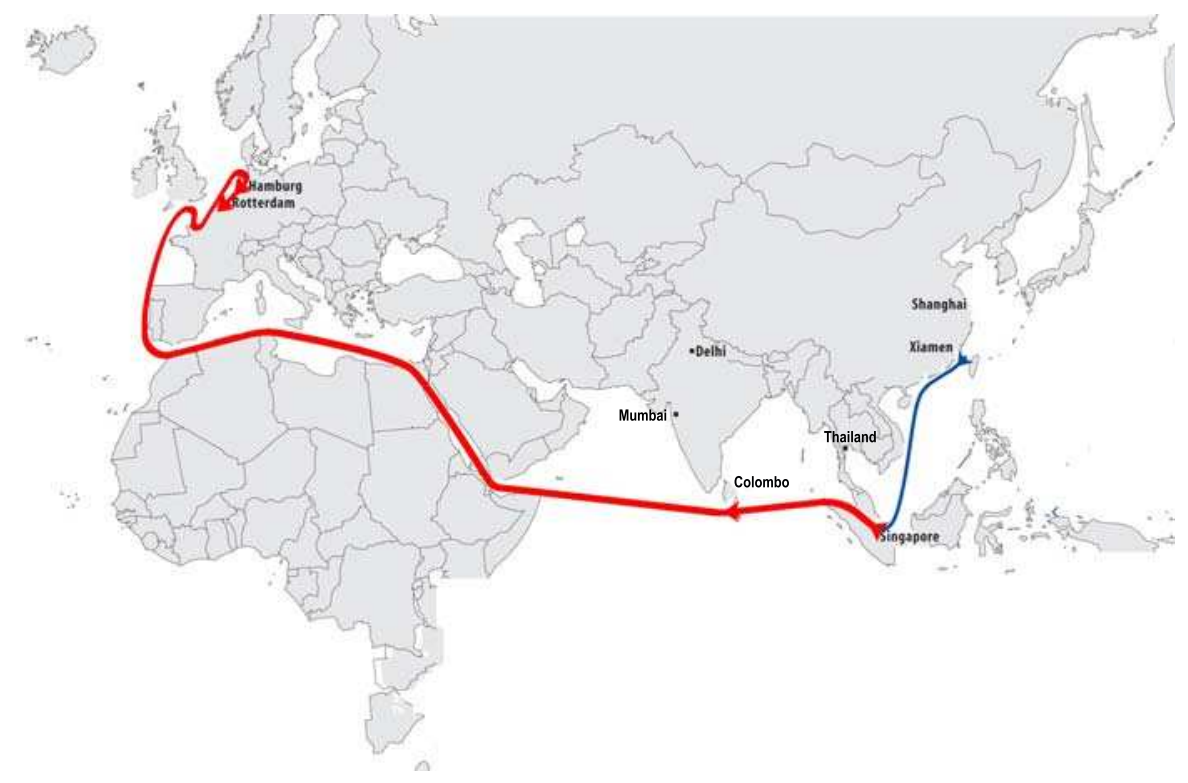

Figure 1: A hypothetical hub-and-spoke maritime network.

In the discrete and competitive model of Marianov et al. (1999), only one single hub link is allowed for any O-D path. For many applications, this is not a realistic model. In particular, for cases where no special structure can be identifie in the cost/distance data - more precisely the triangle inequality is not valid there - optimal structure is not obtained by such formulations. Consider three nodes $a, b$ and $c$ with three links $\|\overline{a b}\|=1,\|\overline{b c}\|=1$ and $\|\overline{a c}\|=3$, where \|..\| is any non-Euclidean norm. Then the optimal solution of a model like (Marianov et al., 1999) in which link $\overline{a c}$ is chosen for transporting concentrated $\mathrm{fl} \mathrm{w}$ between $a$ and $c$ is not the best solution. Rather, there exists another possibility by using two links (i.e. $\overline{a b}$ and $\overline{b c}$ ) and saving 1 unit cost/distance per unit of fl w. Such a solution is not feasible in the model by (Marianov et al., 1999).

The second issue is that even if the cost/distance measures are not taken into account, solutions obtained by these formulations may not be realistic. For instance, let Singapore, Colombo and Rotterdam, as depicted in Figure 1, be three hubs of a maritime transport network of a liner service provider like APL. It is then less attractive for a $\mathrm{fl} \mathrm{w}$ originated from a spoke port Xiamen and destined toward Rotterdam, to be sent to Singapore using spoke-edgeoperating vessels (feeders); then to Colombo by a hub-edge-operating vessel; being unloaded and reloaded to another spoke-edge-operating vessel for shipping to Rotterdam (as a result of (Marianov et al., 1999)). In practice, for the example in Figure 1, the fl w originated from Xiamen after changing the vessel at Singapore remains in a hub-edgeoperating vessel, which departs from Singapore visits Colombo and continues its journey to Rotterdam-even very often after visiting other hub ports like Hamburg, etc. In this way more hub edges can be visited if it is economically attractive.

Wagner (2008) pointed out a few shortcomings in the solution approach proposed by Marianov et al. (1999), but still the aforementioned aspects are not taken into account.

The third issue is that none of the existing models allow for the potential advantages of spoke-to-spoke links being exploited. That means there is no possibility of direct connection between two spokes. This is actually not a realistic and practical assumption in maritime transportation - especially for the network of a small to medium size LSP where the involved economy is smaller compared to the larger size networks.

In this paper, all these issues are taken into account. The proposed mixed integer linear programming model allows multiple stops along the hub-level network as well as direct spoke-spoke connections. Since the model extends the solution space of previous models, the quality of optimal solutions from this model are at least as good as previous approaches. If a general cost structure is assumed then strictly better solutions can be identified

We also assume a multiple allocation scheme, since in liner shipping not only the eastbound and westbound $\mathrm{fl}$ ws from a given spoke port might be allocated to different hub ports but it might also happen that $\mathrm{fl}$ ws in the same 
direction with different destinations are allocated to several hub ports.

Competition between a medium size company $\mathcal{B}$ and a bigger LSP (possibly an alliance) $\mathcal{A}$ that operates on a hub-and-spoke (HS) network can be modeled as the problem of locating $p$ hub ports for $\mathcal{B}$ among all the ports, establishing its connected hub-level network and trying to maximize market shares by meeting some specifi criteria (e.g. minimizing relevant costs and/or time), hence attracting customers from $\mathcal{A}$ as well as new customers from areas not covered by $\mathcal{A}$. To the best of our knowledge, this problem with its particular assumptions and generalizations has not been addressed before in the literature.

This paper is organized as follows: In section 2, we formally state and describe the problem. In section 3, a mathematical model is proposed and its properties are investigated. Section 4 presents a Lagrangian-based decomposition method for obtaining lower bounds. In section 5, we report our computational experiments which indicate that the lower bounds found by the Lagrangian-based relaxation are very promising. The work is concluded in section 6 and further research directions are suggested.

\section{Problem Statement}

The considered problem can formally be described as follows:

Given a finit set of ports $H=\{1,2, \ldots, N\}$, demand between pairs of origin and destination $(O-D)$ ports and a liner transport service provider $\mathcal{A}$ that has already established his HS transportation network on this set of nodes using $q$ hubs, while the rate of offered service and travel time between each pair of ports $i$ and $j$ are known to be $C_{i j}^{\mathcal{A}}$ and $T_{i j}^{\mathcal{A}}$, respectively; the problem seeks to design a hub-and-spoke network of service provider $\mathcal{B}$ with p hubs in total (perhaps overlapping with the hubs of $\mathcal{A})$ so that a weighted sum of the total attractiveness of the services due to the lower rate and shorter service time is maximized.

\subsection{Attraction function}

We introduce an attraction function to indicate the number of customers that can be attracted to the newcomer liner service provider when given a certain time/freight (see Figure 2 for a possible cost attractiveness function). Given $O_{i j}^{c}().\left(O_{i j}^{t}().\right)$ as an attraction function with respect to $\operatorname{cost}($ time $)$ and $C_{i j}\left(T_{i j}\right)$ the O-D transportation cost(time) between $i$ and $j \neq i$, under certain circumstances the attraction function can be reasonably approximated by a step function for given threshold values as follows:

$$
O_{i j}^{c}=\left\{\begin{array}{cc}
O_{i j 1}^{c}, & C_{i j} \leq \beta_{1}^{c} C_{i j}^{\mathcal{A}}, \\
O_{i j 2}^{c}, & \beta_{1}^{c} C_{i j}^{\mathcal{A}}<C_{i j} \leq \beta_{2}^{c} C_{i j}^{\mathcal{A}}, \\
\vdots & \vdots \\
O_{i j F^{c}}^{c}, & \beta_{F^{c}-1}^{c} C_{i j}^{\mathcal{A}}<C_{i j} \leq \beta_{F^{c}}^{c} C_{i j}^{\mathcal{A}} .
\end{array}\right.
$$

and,

$$
O_{i j}^{t}=\left\{\begin{array}{cc}
O_{i j 1}^{t}, & T_{i j} \leq \beta_{1}^{t} T_{i j}^{\mathcal{A}}, \\
O_{i j 2}^{t}, & \beta_{1}^{t} T_{i j}^{\mathcal{A}}<T_{i j} \leq \beta_{2}^{t} T_{i j}^{\mathcal{A}}, \\
\vdots & \vdots \\
O_{i j F^{t}}^{t}, & \beta_{F^{t}-1}^{t} T_{i j}^{\mathcal{A}}<T_{i j} \leq \beta_{F^{t}}^{t} T_{i j}^{\mathcal{A}} .
\end{array}\right.
$$

where, $0<\beta_{1}^{c}<\beta_{2}^{c}<\cdots<\beta_{F^{c}}^{c} \leq 1, \beta_{m}^{c} \in L^{c}$ are the set of values for the cost level, and $0<\beta_{1}^{t}<\beta_{2}^{t}<\cdots<\beta_{F^{t}}^{t} \leq$ $1, \beta_{t}^{m} \in L^{t}$ the set of values for the time level.

There are at least two arguments for using a discretized attraction function: i) in contrast to public transportation where users are more sensitive to small variations in prices/time and their reaction to the prices can be more continuous (perhaps because there is not much work involved in switching to another service provider), in liner transport shippers should take into account additional costs (time and money) involved in switching from their current LSP to another 


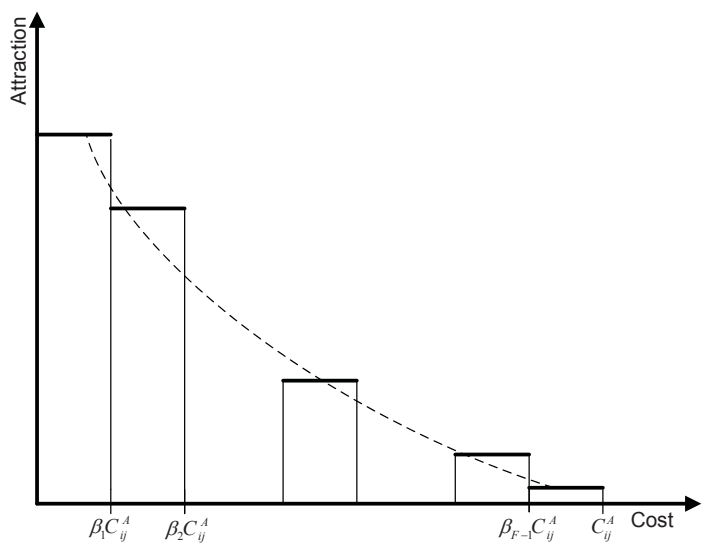

Figure 2: Attraction function approximated by a step-function.

one. Therefore, there are a limited number of threshold values upon which the choice of LSP is based. ii) In the similar situation where both LSPs offer almost the same service fares/time - while the public transport users would prefer to greedily choose the best offer - the liner transport clients would like to avoid monopolies and motivate the competition between the LSPs by opening their markets to both companies. Therefore, there is still hope that the new LSP can obtain share in such markets.

In addition to the aforementioned aspects, it must also be noted that a discretized attraction function removes the computational burden of dealing with a nonlinear objective function.

\section{Mathematical Model}

In this section, we propose a mixed integer programming (MIP) model for the Competitive Hub Location Problem (CMPT-HLP). Variables and parameters are introduced in the following.

We have the following binary variables: For all $i, j, k, l \in H$, we have $h_{i}=1$ if location $i$ is designated as a hub. $x_{i j k l}=1$ if the optimal path from $i$ to $j$ traverses the hub edge $k-l$. Also, $a_{i j k}=1$ if the optimal path from $i$ to $j$ traverses the spoke edge $i-k$ while $i$ is not a hub. $b_{i j k}=1$ if the optimal path from $i$ to $j$ traverses the spoke edge $k-j$ while $j$ is not a hub. In addition, $e_{i j}=1$ if the optimal path from $i$ to $j$ traverses $i-j$ and either $i$ or $j$ is a hub. $s_{i j}=1$, if there is a direct spoke-to-spoke connection between $i$ and $j$ in an optimal solution of the problem. $\gamma_{i j k}=1$ if a transshipment takes place at an intermediate hub $k$ in optimal path from $i$ to $j . \delta_{i j}^{m}=1, m \in\left\{1, \ldots, F^{c}\right\}$ if transportation cost offered by the smaller company between $i$ and $j$ is in $\left(\beta_{m-1}^{c} C_{i j}^{\mathcal{A}}, \beta_{m}^{c} C_{i j}^{\mathcal{A}}\right]$ where $C_{i j}^{\mathcal{A}}$ is the fare offered by $\operatorname{LSP} \mathcal{A}\left(\delta_{i j}^{F^{c}+1}=1\right.$ indicates that the fare offered by $\mathcal{B}$ is more expensive than $\left.\mathcal{A}\right)$.

We have the following linear parameters: $O^{c}\left(\beta_{m}^{c}\right), 0<O^{c}\left(\beta_{m}^{c}\right)<1$ stands for the percentage of market share obtained by the competitor LSP if the cost lies in the interval $\left(\beta_{m-1}^{c} C_{i j}^{\mathcal{A}}, \beta_{m}^{c} C_{i j}^{\mathcal{A}}\right] . \eta_{i j}^{m^{\prime}}=1, m^{\prime} \in\left\{1, \ldots, F^{t}\right\}$ if service time offered by the smaller company between $i$ and $j$ is within $\left(\beta_{m-1}^{t} T_{i j}^{\mathcal{A}}, \beta_{m}^{t} T_{i j}^{\mathcal{A}}\right]$ where $T_{i j}^{\mathcal{A}}$ is the service time offered by LSP $\mathcal{A}\left(\delta_{i j}^{F^{t}+1}=1\right.$ indicates that the travel time offered by $\mathcal{B}$ is more than $\left.\mathcal{A}\right) . O^{t}\left(\beta_{m^{\prime}}^{t}\right), 0<O^{t}\left(\beta_{m^{\prime}}^{t}\right)<1$ stands for the percentage of market share obtained by the competitive LSP if the service time lies in $\left(\beta_{m^{\prime}-1}^{c} T_{i j}^{\mathcal{A}}, \beta_{m^{\prime}}^{c} T_{i j}^{\mathcal{A}}\right]$. $C_{k}^{t r}$ is introduced to represent the transshipment cost per twenty-foot equivalent unit (TEU) at hub port $k$; $t_{i j}$ stands for the travel time between $i$ and $j$ and $T_{i j k}^{t r}$ depicts the transit time - which is a priori known according to the schedules-for the $\mathrm{fl} \mathrm{w}$ from $i$ to $j$ at hub port $k$ and; $C_{k}^{h}$ is the holding cost incurred proportional to the length of time that a single container is hosted by hub port $k$.

The considered objective function is a convex combination of attractiveness in terms of both cost and time. The weight $\lambda$ is set by the decision maker (DM) and indicates the relative preference given by DM to each function.

The transportation cost/time for a given $\mathrm{fl} \mathrm{w}$ with origin $i$ and destination $j$ accounts for the sum of, (i) the cost/time of sending the $\mathrm{fl} \mathrm{w}$ from $i$ to the firs hub node, (ii) the transshipment cost/time (including unloading, 
holding, and re-loading) at the firs hub port, (iii) cost/time of traversing one or more hub edges discounted by the factor $\alpha(0<\alpha<1)$ introduced in Section 1, (iv) transshipment cost/time at the last hub port and, (v) the cost/time of transportation on the last spoke edge.

\subsection{CMPT-HLP}

The mathematical model for the hub location problem in a competitive environment (CMPT-HLP) can now be formulated as:

$$
\begin{aligned}
& \operatorname{Max} \lambda \sum_{i} \sum_{j \neq i} \sum_{m=1}^{F^{c}} O_{i j m}^{c}\left(\beta_{m}^{c}\right) \delta_{i j}^{m}+(1-\lambda) \sum_{i} \sum_{j \neq i} \sum_{m^{\prime}=1}^{F^{t}} O_{i j m^{\prime}}^{t}\left(\beta_{m^{\prime}}^{t}\right) \eta_{i j}^{m^{\prime}} \\
& \text { s.t. } \quad \sum_{k} h_{k}=p \\
& \sum_{l \neq i} x_{i j l l}+\sum_{l \neq i, j} a_{i j l}+e_{i j}+s_{i j}=1, \\
& \sum_{l \neq j} x_{i j l j}+\sum_{l \neq i, j} b_{i j l}+e_{i j}+s_{i j}=1 \text {, } \\
& \sum_{l \neq k, i} x_{i j k l}+b_{i j k}=\sum_{l \neq k, j} x_{i j l k}+a_{i j k} \\
& x_{i j k l}+x_{i j l k} \leq h_{k}, \\
& x_{i j k l}+x_{i j l k} \leq h_{l}, \\
& \sum_{l \neq k} x_{k j k l} \leq h_{k}, \\
& \sum_{k \neq l} x_{i l k l} \leq h_{l} \\
& a_{i j k}+\sum_{l \neq j, k} x_{i j l k} \leq h_{k}, \\
& b_{i j k}+\sum_{l \neq k, i} x_{i j k l} \leq h_{k}, \\
& e_{i j}+2 x_{i j i j}+\sum_{l \neq j, i} x_{i j i l}+\sum_{l \neq i, j} x_{i j l j} \leq h_{i}+h_{j}, \\
& \sum_{k, l \neq k} \alpha C_{k l} x_{i j k l}+\sum_{k \neq i, j} C_{i k} a_{i j k}+\sum_{k \neq i, j} C_{k j} b_{i j k}+C_{i j}\left(e_{i j}+s_{i j}\right) \\
& +\sum_{k \neq i, j}\left(2 C_{k}^{t r}+T_{i j k}^{t r} C_{k}^{h}\right) \gamma_{i j k} \leq C_{i j}^{\mathcal{A}} \sum_{m \in F^{c}} \beta_{m}^{c} \delta_{i j}^{m}+M \delta_{i j}^{F^{c}+1}, \\
& \forall i, j \neq i, \\
& \forall i, j \neq i, \\
& \forall i, j \neq i, \\
& \forall i, j \neq i, k \neq i, j, \\
& \forall i, j \neq i, k, l>k, \\
& \forall i, j \neq i, k, l>k \text {, } \\
& \forall j, k \neq j, \\
& \forall i, l \neq i, \\
& \forall i, j \neq i, k \neq i, j, \\
& \forall i, j \neq i, k \neq i, j, \\
& \forall i, j \neq i, \\
& \sum_{m=1}^{F^{c}+1} \delta_{i j}^{m}=1 \\
& \sum_{k, l \neq k} t_{k l} x_{i j k l}+\sum_{k \neq i, j} t_{i k} a_{i j k}++\sum_{k \neq i, j} t_{k j} b_{i j k}+t_{i j}\left(e_{i j}+s_{i j}\right) \\
& +\sum_{k \neq i, j} \gamma_{i j k} T_{i j k}^{t r} \leq C_{i j}^{\mathcal{A}} \sum_{m \in F^{T}} \beta_{m^{\prime}}^{t} \eta_{i j}^{m^{\prime}}+M \eta_{i j}^{F^{T}+1} \\
& \forall i, j \neq i, \\
& \sum_{m^{\prime}=1}^{F^{T}+1} \eta_{i j}^{m^{\prime}}=1 \\
& \gamma_{i j k} \leq h_{k} \text {, }
\end{aligned}
$$




$$
\begin{array}{lr}
\gamma_{i j k} \leq a_{i j k}+b_{i j k}, & \forall i, j \neq i, k \neq i, j, \\
\gamma_{i j k} \leq 1-s_{i j}, \gamma_{i j k} \leq 1-e_{i j}, & \forall i, j \neq i, k \neq i, j, \\
\gamma_{i j k} \geq a_{i j k}, \gamma_{i j k} \geq b_{i j k}, & \forall i, j \neq i, k \neq i, j, \\
e_{i j} \leq 2-\left(h_{i}+h_{j}\right), & \forall i, j \neq i, \\
s_{i j} \leq 1-h_{i}, s_{i j} \leq 1-h_{j}, & \forall i, j, \\
a_{i j k} \leq 1-h_{i}, & \forall i, j \neq i, k \neq i, j, \\
b_{i j l} \leq 1-h_{j}, & \forall i, j \neq i, l \neq i, j, \\
x_{i j k l}, y_{k l}, h_{k}, a_{i j k}, b_{i j k}, e_{i j}, \eta^{m^{\prime}}, \delta^{m} \in\{0,1\} . &
\end{array}
$$

The objective (3) is to maximize the weighted sum of market shares due to cost and time. Constraint (4) determines the number of hub nodes to be established. Constraints (5)-(7) are the $\mathrm{fl} \mathrm{w}$ conservation constraints. The constraints (8)-(9) ensure that the traversed links in the hub-level network have both end-points as hub nodes. In ( 10) (resp. (11)) it is ensured that only a f ow with origin (destination) in a hub is allowed to select a hub edge to depart from the origin (arrive to the destination). Any fow from $i$ to $j$ which enters to (depart from) a node other than $i$ and $j$ should be a hub node. This is ensured by (12) (resp. (13)). The choice of edges on the path from origin $i$ to destination $j$ depends on whether $i$ and $j$ both, none, or just one of them is a hub node. This is ensured by (14). Constraints (15) determine the level of transportation cost that $\mathcal{B}$ achieves. That means, for every $i, j \neq i$ either the transport cost lies within two consecutive thresholds which makes the constraint become active or transportation cost exceeds that of $\mathcal{A}, \delta_{i j}^{F^{c}+1}$ becomes one, and the constraint becomes redundant. Therefore the service provider does not attract customers with respect to the cost on the given $i$ to $j$ path. This cost includes on-shore and off-shore costs. Constraints (16) ensure that either one level is met or the fare is more expensive. Analogously, (17) and (18) stands for the service time level. Existence of any stopover on any hub node along an origin-destination pair is determined by constraints (19)-(22). The end-points of links are controlled by (23)-(26).

\section{Solution Method}

Preliminary computational experiments indicate that (CMPT-HLP) is a very challenging problem where even small sized instances cannot be solved in reasonable time by standard MIP solvers. Table 1 shows that even for $n=10$ and objective function weights $\lambda \in\{0.4,0.6\}$, CPLEX fails to solve some cases of the problem to optimality.

We propose a Lagrangian decomposition method, combined with additional procedures to calculate primal bounds, obtaining tight bounds for the optimal solutions.

\subsection{Lagrangian relaxation}

Lagrangian relaxation for solving integer programming problems was firs proposed in Fisher (1981, 2004). The idea behind this method is to relax complicating constraints by penalizing the objective function upon violation of these constraints. The relaxed problem is expected to be easier to solve than the original problem and provides a dual bound on the optimal solution of the problem. See Guignard (2003) for a comprehensive survey of the method.

A few Lagrangian relaxation based methods for solving various variants of HLPs have shown promising results. Among such papers we refer to Aykin (1994), Pirkul and Schilling (1998), Sasaki and Fukushima (2003), Marín (2005b), Elhedhli and $\mathrm{Hu}$ (2005) and the recent paper Contreras et al. (2008).

Lagrangian relaxing constraints (5), (6), (7), (10),(11), (12), (13) and (14) using the multipliers $u_{i j}^{1}, u_{i j}^{2}, u_{i j k}^{3} \in \mathbb{R}$, and $u_{i j}^{4}, u_{i j}^{5}, u_{i j k}^{6}, u_{i j k}^{7}, u_{i j k}^{8} \in \mathbb{R}_{+}$, respectively. In this way the relaxed model (LRX-CMPT-HLP) becomes:

$$
\begin{aligned}
& L\left(u_{1}, u_{2}, u_{3}, u_{4}, u_{5}, u_{6}, u_{7}, u_{8}\right)= \\
& \max \lambda \sum_{i} \sum_{j \neq i} \sum_{m=1}^{F^{c}} O_{i j m}^{c}\left(\beta_{m}^{c}\right) \delta_{i j}^{m}+(1-\lambda) \sum_{i} \sum_{j \neq i} \sum_{m^{\prime}=1}^{F^{t}} O_{i j m}^{t}\left(\beta_{m^{\prime}}^{t}\right) \eta_{i j}^{m^{\prime}}+ \\
& \sum_{i, j \neq i}-u_{i j}^{1}\left(\sum_{l \neq i} x_{i j i l}+\sum_{l \neq i, j} a_{i j l}+e_{i j}+s_{i j}-1\right)+\sum_{i, j \neq i}-u_{i j}^{2}\left(\sum_{l \neq j} x_{i j l j}+\sum_{l \neq i, j} b_{i j l}+e_{i j}+s_{i j}-1\right)+
\end{aligned}
$$




$$
\begin{aligned}
& \sum_{i, j, k \neq i, j}-u_{i j k}^{3}\left(\sum_{l \neq k, i} x_{i j k l}+b_{i j k}-\sum_{l \neq k, j} x_{i j l k}-a_{i j k}\right)+ \\
& \sum_{j, k \neq j}-u_{j k}^{4}\left(\sum_{l \neq k} x_{k j k l}-h_{k}\right)+\sum_{i, l \neq i}-u_{i l}^{5}\left(\sum_{k \neq l} x_{i l k l}-h_{l}\right)+ \\
& \sum_{i, j, k \neq i, j}-u_{i j k}^{6}\left(a_{i j k}+\sum_{l \neq j, k} x_{i j l k}-h_{k}\right)+\sum_{i, j, k \neq i, j}-u_{i j k}^{7}\left(b_{i j k}+\sum_{l \neq k, i} x_{i j k l}-h_{k}\right)+ \\
& \sum_{i, j \neq i}-u_{i j}^{8}\left(e_{i j}+2 x_{i j i j}+\sum_{l \neq j, i} x_{i j i l}+\sum_{l \neq i, j} x_{i j l j}-h_{i}-h_{j}\right)
\end{aligned}
$$

s.t. $\quad(4),(8),(9),(15)-(27)$

We solve the Lagrangian dual problem

$$
Z_{D}=\min _{\left\{\begin{array}{l}
u_{1}, u_{2}, u_{3} \in \mathbb{R}, \\
u_{4}, u_{5}, u_{6}, u_{7}, u_{8} \geq 0
\end{array}\right\}} L\left(u_{1}, u_{2}, u_{3}, u_{4}, u_{5}, u_{6}, u_{7}, u_{8}\right),
$$

using the subgradient procedure outlined in Algorithm 1. Step 1 to 8 is repeated for iteration $k=1,2, \ldots$ until the stopping condition is met.

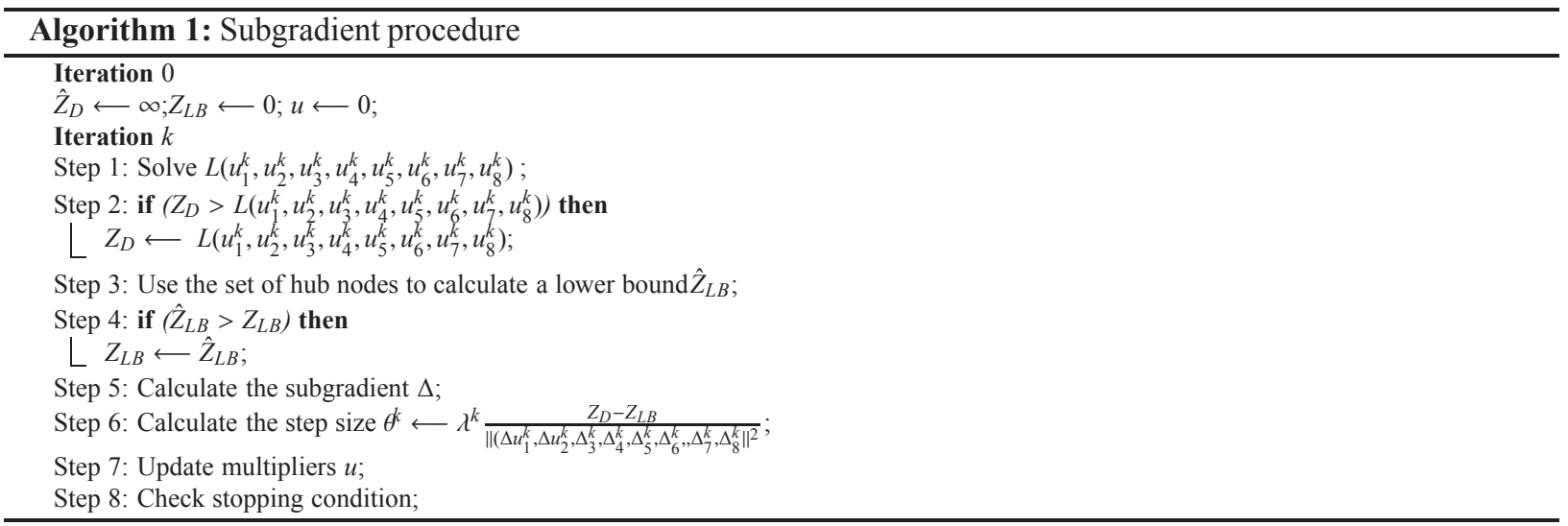

\subsubsection{Reduction heuristic}

The (LRX-CMPT-HLP) contains a large number of columns, many of which do not appear in the optimal solution. Several of these columns can be removed in a pre-processing phase. After re-writing the objective function of (LRXCMPT-HLP) in terms of the original variables, we identify the case where variables can be omitted and reduce the size of the problem.

The (LRX-CMPT-HLP) can be formulated as the following MIP model:

$\operatorname{Max}$

$$
\begin{aligned}
& \lambda \sum_{i} \sum_{j \neq i} \sum_{m=1}^{F^{c}} O_{i j}^{c}\left(\beta_{c}^{m}\right) \delta_{i j}^{m}+(1-\lambda) \sum_{i} \sum_{j \neq i} \sum_{m^{\prime}=1}^{F^{t}} O_{i j}^{t}\left(\beta_{t}^{m^{\prime}}\right) \eta_{i j}^{m}+ \\
& \sum_{i, j \neq i}\left(-u_{i j}^{1}-u_{i j}^{2}-u_{i j}^{8}+\right) e_{i j}+\sum_{i, j \neq i}\left(-u_{i j}^{1}-u_{i j}^{2}\right) s_{i j}+ \\
& \sum_{i, j \neq i} \sum_{k \neq i, j}\left(-u_{i j}^{1}+u_{i j k}^{3}-u_{i j k}^{6}\right) a_{i j k}+\sum_{i, j \neq i} \sum_{k \neq i, j}\left(-u_{i j}^{2}-u_{i j k}^{3}-u_{i j k}^{7}\right) b_{i j k}+
\end{aligned}
$$




$$
\begin{aligned}
& \sum_{i, j \neq i}\left(-u_{i j}^{1}-u_{i j}^{2}-u_{j i}^{4}-u_{i j}^{5}-2 u_{i j}^{8}\right) x_{i j i j}+ \\
& \sum_{i, j \neq i} \sum_{k \neq i, j}\left(-u_{i j}^{2}-u_{i j k}^{3}-u_{i j}^{5}-u_{i j k}^{7}-u_{i j}^{8}\right) x_{i j k j}+ \\
& \sum_{i, j \neq i} \sum_{k \neq i, j} \sum_{l \neq i, j}\left(-u_{i j k}^{3}+u_{i j l}^{3}-u_{i j l}^{6}-u_{i j k}^{7}\right) x_{i j k l}+ \\
& \sum_{k}\left(\sum_{i \neq k} \sum_{j \neq i, k}\left(u_{i j k}^{6}+u_{i j k}^{7}\right)+\sum_{j \neq k}\left(u_{j k}^{8}+u_{k j}^{8}+u_{j k}^{4}+u_{k j}^{5}\right)\right) h_{k}+ \\
& \left(\sum_{i, j \neq i} u_{i j}^{1}+\sum_{i, j \neq i} u_{i j}^{2}\right)
\end{aligned}
$$

Then we introduce:

$$
\begin{array}{ll}
E_{i j}=-u_{i j}^{1}-u_{i j}^{2}-u_{i j}^{8}, & \forall i, j \neq i, \\
S_{i j}=-u_{i j}^{1}-u_{i j}^{2}, & \forall i, j \neq i, \\
A_{i j k}=-u_{i j}^{1}+u_{i j k}^{3}-u_{i j k}^{6}, & \forall i, j \neq i, k \neq i, j, \\
B_{i j k}=-u_{i j}^{2}-u_{i j k}^{3}-u_{i j k}^{7}, & \forall i, j \neq i, k \neq i, j,
\end{array}
$$

For all $i, j \neq i, k \neq l$,

$$
C_{i j k l}^{\prime}= \begin{cases}-u_{i j}^{1}-u_{i j}^{2}-u_{j i}^{4}-u_{i j}^{5}-2 u_{i j}^{8}, & \forall i=k, j=l, \\ -u_{i j}^{2}-u_{i j k}^{i}-u_{i j}^{5}-u_{i j k}^{7}-u_{i j}^{8}, & \forall i \neq k, j=l, \\ -u_{i j}^{1}+u_{i j l}^{3}-u_{j i}^{4}-u_{i j l}^{6}-u_{i j}^{8}, & \forall i=k, j \neq l, \\ -u_{i j k}^{i}+u_{i j l}^{3}-u_{i j l}^{6}-u_{i j k}^{7}, & \forall i \neq k, j \neq l .\end{cases}
$$

We can now use the following reduction rules to eliminate a subset of variables from the formulation.

Proposition 1. if $E_{i j} \leq 0$ for any $i, j$ then $e_{i j}=0$.

Proposition 2. if $S_{i j} \leq 0$ for any $i, j$ then $s_{i j}=0$.

Proposition 3. if $A_{i j k} \leq 0$ for any $i, j, k$ then $a_{i j k}=0$.

Proposition 4. if $B_{i j k} \leq 0$ for any $i, j, k$ then $b_{i j k}=0$.

Proposition 5. if $C_{i j k l}^{\prime} \leq 0$ for any $i, j, k$, l then $x_{i j k l}=0$. Moreover, if $C_{i j k l}^{\prime}>0$ and $C_{i j l k}^{\prime}>0$ :

$i$ if $C_{i j k l}^{\prime}<C_{i j l k}^{\prime}$ then $x_{i j k l}=0$ and,

ii if $C_{i j k l}^{\prime}>C_{i j l k}^{\prime}$ then $x_{i j l k}=0$.

\subsection{Primal bound}

The set of hub nodes plays the most important role in any optimal solution to (CMPT-HLP). In fact, once the location of the hub nodes is selected the remaining problem is much easier to solve, in particular if it can be decomposed for each O-D pair. More precisely, once the hub nodes are know, constraint ( 4) will be dropped from (CMPT-HLP) and constraints (8), (9) and (15)-(27) are decomposable for every O-D pair to $n(n-1)$ sub-problem.

In summary, any optimal solution to the LR model is an upper bound on the optimal solution of the original problem, and the found set of hub nodes can be used to obtain a feasible solution to the original problem. This saves us from using additional heuristics or local search methods to obtain lower bounds. 


\section{Computational Results}

In this section we describe the presented Lagrangian algorithm in more details and compare its performance with CPLEX. All results have been obtained on an Intel(R) Core(TM)2 Quad CPU $2.66 \mathrm{GHz}$ with $4 \mathrm{~Gb}$ RAM.

It should be noted that due to the particular (Euclidean) cost structure of the instances in the classical hub location problem data ${ }^{2}$, we have generated a new set of instances based on historic regional data from an anonymous liner service provider.

\subsection{Lagrangian relaxation}

In order to examine the performance of the presented Lagrangian algorithm and the quality of the obtained solutions, we only consider instances of practical size in liner shipping for which an optimal solution has been obtained by ILOG-CPLEX.

In our subgradient procedure, the parameter $\lambda$ is initially set to 2 . If the upper bound is not improved for three consecutive iterations, $\lambda$ is divided by 1.5. The relaxed problem in Step 1 is solved by CPLEX. Preliminary computational experiments indicate that it is not necessary at this point to solve the LR problem to optimality. Sometimes, this does decelerate the convergence rate of the algorithm especially in the very early iterations. Therefore, we set the solver parameter IloCplex: :EpGap to a big number and depending on the machine configuratio also IloCplex: :TiLim to values among 10-30. By doing so the algorithm shows a much faster convergence. It also helps the primal bound generator procedure to obtain good lower bounds in the very early iterations. To avoid repeating expensive calculations of the primal bound for the same set of hub nodes, we store the objective values and retrieve them, if any, before invoking the solver. Nevertheless, since the LR is not solved to optimality it may happen that at some iteration the upper bound reduces to a value less than the lower bound. In such cases we add another functionality to identify the opened hubs in such cases: Assume that $I^{h}$ is the index set of hub nodes we add the following combinatorial cuts and re-optimize.

$$
\sum_{i \in I^{h}} h_{i} \leq p-1
$$

These cuts are removed before going to the next iteration.

\subsection{A practical size instance}

This instance has been constructed by clustering smaller ports in the considered region and represent them by a single port. The same instance can be de-clustered to represent larger size instances at the cost of a higher computational burden. The performance of our Lagrangian approach is compared against the standard solver on all the instances of size 10 for which the optimal solution are known from CPLEX. For $n=10$, the geographical distribution of the nodes is depicted in Figure 3.

We have chosen $\left(\beta_{m}^{c}, O_{i j m}^{c}\left(\beta_{m}^{c}\right)\right) \in\{(0.65,0.4),(0.75,0.3),(0.9,0.2)\} \forall i, j \neq i, m \in\left\{1, \ldots F^{c}\right\}$ and $\left(\beta_{m}^{t}, O_{i j m}^{t}\left(\beta_{m}^{t}\right)\right) \in$ $\{(0.25,0.6),(0.50,0.5),(0.75,0.4),(1,0.0)\} \forall i, j \neq i, m \in\left\{1, \ldots F^{T}\right\}$.

We solve this instance $(n=10, q=2)$ where $q$ is the number of hub nodes in the service network of $\mathcal{A}$ and for discount factors $\alpha \in\{0.6,0.75,0.9\}$, and $p \in\{2,4\}$ where $p$ is the number of hubs aimed to be opened by $\mathcal{B}$. Tables 1 and 2 report the results. For $(n=10, q=4)$ the model is solved for $p=4$ and Table 3 summarizes the results.

In each of the tables we have divided the rows into three blocks with respect to the values of $\alpha \in\{0.6,0.75,0.9\}$ in the firs column. In each block f ve different values of $\lambda \in\{0,0.2,0.4,0.6,0.8,1\}$ are considered. The third column 'hub' reports the optimal hubs. Fourth column 'obj.val' reports the optimal objective value of the instance, if obtained, and the best objective value when the solver failed to continue (superscript indicates the reported gap). Next column reports the root node gap 'r.n.g' for each instance, reported by CPLEX. The column ' ${ }^{C P L E X}$ ' reports the computational time in seconds required to obtain the optimal solution, if successful. In the seventh column 'nr.iter' we report the number of subgradient iterations in our Lagrangian algorithm. The next column 'gap ${ }^{L R}$, reports the quality of the solution obtained in terms of relative gap in percent between the lower and upper bounds. The next column ' $t{ }^{L R}$,

${ }^{2}$ OR-Library (www.people.brunel.ac.uk/ mastjjb/jeb/info.html) 


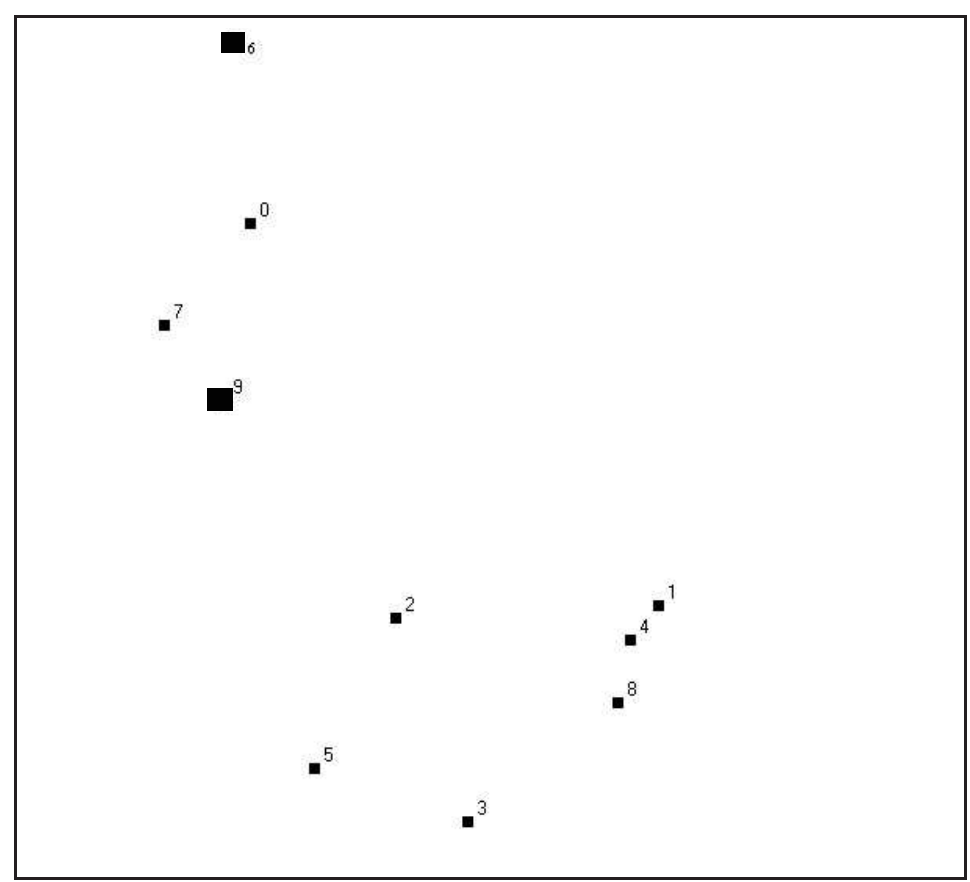

Figure 3: Geographical distribution of the network with $n=10$ and $q=2$ hubs located at ports 6 and 9.

reports the computational time in seconds spent by the Lagrangian method before meeting the stopping criteria. The last column ' $t_{L B}^{C P L E X}$, reports the required time in seconds for CPLEX to obtain a solution with a similar quality as our approach.

Table 1: Computational results for a random instance with $n=10, q=2, p=2$.

\begin{tabular}{|c|c|c|c|c|c|c|c|c|c|}
\hline \multirow[b]{2}{*}{$\alpha$} & \multirow[b]{2}{*}{$\lambda$} & \multirow[b]{2}{*}{ hub } & \multicolumn{3}{|c|}{ CPLEX } & \multicolumn{3}{|c|}{ LR } & \multirow{2}{*}{$\frac{\text { Comparison }}{t_{L B}^{C P L E X}(\mathrm{sec} .)}$} \\
\hline & & & obj.val & r.n.g. $(\%)$ & $t^{C P L E X}$ (sec.) & nr. iter & gap $^{L R}$ & $t^{L R}(\mathrm{sec})$. & \\
\hline \multirow[t]{6}{*}{0.6} & 0 & $\{7,8\}$ & 70434.30 & 47.18 & 216.11 & 86 & 1.12 & 1305.03 & 192.36 \\
\hline & 0.2 & $\{-,-\}$ & $61117.26^{21.57 \%}$ & 90.29 & - & 15 & 0.40 & 231.13 & - \\
\hline & 0.4 & $\{-,-\}$ & $51296.66^{50.85 \%}$ & 128.22 & - & 17 & 0.30 & 277.88 & - \\
\hline & 0.6 & $\{3,9\}$ & 43845.40 & 176.34 & 14991.70 & 33 & 1.39 & 659.44 & 14851.12 \\
\hline & 0.8 & $\{6,9\}$ & 41559.35 & 254.23 & 14906.61 & 13 & 0.01 & 186.06 & 14855.65 \\
\hline & 1 & $\{6,9\}$ & 54681.40 & 321.44 & 15064.44 & 32 & 2.25 & 477.26 & 13462.25 \\
\hline \multirow[t]{6}{*}{0.75} & 0 & $\{7,8\}$ & 70264.10 & 49.16 & 355.04 & 75 & 3.69 & 1227.25 & 302.6 \\
\hline & 0.2 & $\{-,-\}$ & $60996.90^{13.17 \%}$ & 78.69 & - & 14 & 0.21 & 216.17 & - \\
\hline & 0.4 & $\{-,-\}$ & $52173.26^{38.05 \%}$ & 114.44 & - & 34 & 0.89 & 599.20 & - \\
\hline & 0.6 & $\{3,9\}$ & 44543.80 & 148.89 & 14803.39 & 12 & 0.78 & 185.88 & 14732.25 \\
\hline & 0.8 & $\{2,6\}$ & 41657.30 & 209.41 & 14857.24 & 32 & 3.95 & 703.66 & 14652.01 \\
\hline & 1 & $\{6,9\}$ & 48083.40 & 243.72 & 14865.44 & 35 & 4.09 & 523.42 & 13258.78 \\
\hline \multirow[t]{6}{*}{0.9} & 0 & $\{7,8\}$ & 67008.80 & 54.27 & 207.33 & 54 & 9.46 & 816.94 & 152.26 \\
\hline & 0.2 & $\{-,-\}$ & $58194.32^{15.35 \%}$ & 81.93 & - & 39 & 4.46 & 902.06 & - \\
\hline & 0.4 & $\{-,-\}$ & $49951.28^{38.68 \%}$ & 117.24 & - & 20 & 0.74 & 338.27 & - \\
\hline & 0.6 & $\{2,9\}$ & 43112.5 & 145.36 & 15105.3 & 34 & 4.50 & 690.74 & 13028.25 \\
\hline & 0.8 & $\{2,6\}$ & 43745.1 & 173.25 & 14980.45 & 31 & 0.17 & 573.52 & 14962.11 \\
\hline & 1 & $\{6,9\}$ & 54681.4 & 225.3 & 14890.6 & 34 & 0.28 & 507.41 & 14802.14 \\
\hline
\end{tabular}

When we only aim at minimizing the travel times against those of $\mathcal{A}$ (i.e. $\lambda=0$ ), the hub nodes are more oriented toward the central part of geographical area. For $\lambda=1$ the aim is to compete for cheaper service costs rather than transportation times. For the two cases of $\lambda \in\{0.2,0.4\}$ the standard solver failed in obtaining an optimal solution (this behavior is observed for instances with $n=10$ for all different parameters $q$ and $\lambda$ ) and in such cases the best 
know solutions are stated (with the reported gaps). In the fourth column, one observes that in general the root node gaps are quite high. As $\lambda$ increase the gaps grow dramatically. We set a time limit of 6 hrs and report 'LIMIT' if the computational time exceeds this limit.

In the sixth column 'nr.iter' one observes that only in very rare cases, the number of iterations exceeds 50 and this mainly occurs for $\lambda=1$ (in Table 3 for $\alpha=0.9$ ). The gaps reported in column gap ${ }^{L R}$ of Table 1 and Table 2 are very small, often less than 1 percent. In some cases of $\lambda=0,1$ the gap jumps to higher values. This is due to the fact that when the objective function does not depend on $\eta_{i j}^{m^{\prime}}($ for $\lambda=1)$ or $\delta_{i j}^{m}($ for $\lambda=0$ ) then due to the big-M nature of constraints (15) and (17) the formulation becomes rather loose (there will be hub-level configuration where the heuristic returns solutions with bad objective value). In Table 3 the gaps are still acceptable and in most cases less than 10 percent except for two outliers cases.

Generally - except for a few cases where the optimal solution is not known - the solution is found faster by our approach than by CPLEX, even in those cases where the solution quality is not very high. Very often, our approach is at least one order of magnitude faster than CPLEX for findin solutions of similar quality.

Table 2: Computational results for a random instance with $n=10, q=2, p=4$.

\begin{tabular}{|c|c|c|c|c|c|c|c|c|c|}
\hline \multirow[b]{2}{*}{$\alpha$} & \multirow[b]{2}{*}{$\lambda$} & \multirow[b]{2}{*}{ hub } & \multicolumn{3}{|c|}{ CPLEX } & \multicolumn{3}{|c|}{ LR } & \multirow{2}{*}{$\frac{\text { Comparison }}{t_{L B}^{C P L E X}(\mathrm{sec} .)}$} \\
\hline & & & obj.val & r.n.g. $(\%)$ & $t^{C P L E X}(\mathrm{sec})$. & nr.iter & $\operatorname{gap}^{L R}$ & $t^{L R}(\mathrm{sec})$. & \\
\hline \multirow[t]{6}{*}{0.6} & 0 & $\{7,8,6,9\}$ & 76823.5 & 47.25 & 320.1 & 26 & 87.85 & 385.63 & 85.89 \\
\hline & 0.2 & $\{-,-,-,-\}$ & $65996.3^{25.45 \%}$ & 110.11 & 14051.3 & 27 & 0.89 & 407.87 & 13985.25 \\
\hline & 0.4 & $\{-,-,-,-\}$ & $59573.2^{35.1 \%}$ & 108.63 & - & 28 & 0.39 & 425.99 & - \\
\hline & 0.6 & $\{2,8,6,9\}$ & 53518 & 150.25 & - & 34 & 0.18 & 576.96 & - \\
\hline & 0.8 & $\{2,8,6,9\}$ & 58163.3 & 167.64 & 15182.12 & 29 & 0.01 & 447.62 & 18182.10 \\
\hline & 1 & $\{2,8,6,9\}$ & 74023 & 172.48 & 14992.23 & 16 & 44.69 & 231.10 & 10985.25 \\
\hline \multirow[t]{6}{*}{0.75} & 0 & $\{7,8,6,9\}$ & 76653.3 & 55.14 & 452.14 & 17 & 89.34 & 246.89 & 23.52 \\
\hline & 0.2 & $\{-,-,-,-\}$ & $67219^{33.48 \%}$ & 71.29 & - & 37 & 0.28 & 575.84 & - \\
\hline & 0.4 & $\{-,-,-,-\}$ & $58112^{12.52 \%}$ & 105.23 & - & 51 & 1.42 & 801.19 & - \\
\hline & 0.6 & $\{4,8,6,9\}$ & 52311.9 & 126.59 & 11007.5 & 48 & 0.80 & 796.17 & 10955.02 \\
\hline & 0.8 & $\{4,8,6,9\}$ & 51869.7 & 199.69 & 11999.11 & 36 & 0.96 & 573.90 & 11842.25 \\
\hline & 1 & $\{4,8,6,9\}$ & 62684.8 & 225.27 & 13014.01 & 16 & 72.25 & 231.61 & 1577.23 \\
\hline \multirow[t]{6}{*}{0.9} & 0 & $\{7,8,6,9\}$ & 73398 & 49.47 & 412.52 & 42 & 79.52 & 631.98 & 45.14 \\
\hline & 0.2 & $\{-,-,-,-\}$ & $64097.4^{10.14 \%}$ & 55.12 & - & 49 & 0.01 & 764.83 & - \\
\hline & 0.4 & $\{-,-,-,-\}$ & $55854.4^{55.12 \%}$ & 89.45 & - & 63 & 0.75 & 1123.30 & - \\
\hline & 0.6 & $\{4,8,6,9\}$ & 47120.9 & 155.45 & 11702.48 & 43 & 0.93 & 698.10 & 11659.41 \\
\hline & 0.8 & $\{4,8,6,9\}$ & 49712.2 & 175.66 & 11892.51 & 52 & 1.75 & 925.07 & 10552.32 \\
\hline & 1 & $\{4,8,6,9\}$ & 61705.6 & 213.58 & 13665.5 & 16 & 72.61 & 231.86 & 5136.2 \\
\hline
\end{tabular}

\subsection{Larger sized instances}

The liner shipping sub-network of our industrial partner consists of 72 ports. A part of this set are ports which have minor demands and are geographically close to each other. Therefore they can be clustered as a single spoke node. None of the ports in each cluster have an infrastructure justifying that they are considered as hub ports. Among the ports with higher demands, again some cannot be considered as hubs. This is either because of the nature of business or due to the lack of infrastructure. However, the output of the clustering will have at most 28 total ports from which at most 10 can potentially be hub ports.

For larger instances, CPLEX was not able to solve the MIP formulation within the time limit of $6 \mathrm{hrs,} \mathrm{and} \mathrm{hence} \mathrm{the}$ optimal solutions are not know. Thus, we only report the quality of the heuristic solutions found and the corresponding computational time. We consider instances with $n=15, q=3$ and $p \in\{3,5\}$ and with $n=20, q=5$ and $p \in\{5,7\}$. In Tables 4-5 we report for $\alpha \in\{0.6,0.75,0.9\}$, and $\lambda \in\{0,0.25,0.5,0.75,1\}$, the number of iterations in the subgradient algorithm in the second column. Next column reports the gap of the obtained solution, and the fourth one reports the computational times. As a stop criterion we have chosen a time limit of $6 \mathrm{hrs}$ for the subgradient algorithm. Wherever the time limit is met, the number of iterations and the relative gap calculated as $(U B-L B) / L B$ is reported. If the time limit is exceeded the last column says 'LIMIT'.

It is generally seen in the following tables that for $p=q$ the problems become harder to solver. When the discount rate $\alpha$ is increased the average gap as well as the extreme case gap generally grows. However, this trend is hardly 
Table 3: Computational results for a random instance with $n=10, q=4, p=4$.

\begin{tabular}{|c|c|c|c|c|c|c|c|c|c|}
\hline & & & & CPLEX & & & LR & & Comparison \\
\hline$\alpha$ & $\lambda$ & hub & obj.val & r.n.g. $(\%)$ & $t^{C P L E X}(\mathrm{sec})$. & nr.iter & $\mathrm{gap}^{L R}$ & $t^{L R}(\mathrm{sec})$. & $t_{L B}^{C P L E X}(\mathrm{sec})$. \\
\hline \multirow[t]{6}{*}{0.6} & 0 & $\{4,6,7,8\}$ & 88164.2 & 65.12 & 780.88 & 17 & 62.77 & 247.178 & 205.29 \\
\hline & 0.2 & $\{-,-,-,-\}$ & $76249.8^{60.01 \%}$ & 125.28 & - & 54 & 4.67 & 823.097 & - \\
\hline & 0.4 & $\{-,-,-,-\}$ & $62300.4^{22.85 \%}$ & 145.70 & - & 60 & 3.93 & 917.796 & - \\
\hline & 0.6 & $\{3,5,7,9\}$ & 52139.7 & 144.01 & 13782.07 & 35 & 12.63 & 551.25 & 8236.25 \\
\hline & 0.8 & $\{0,2,4,5\}$ & 43911.5 & 189.33 & 14015.45 & 70 & 4.10 & 1101.79 & 13045.82 \\
\hline & 1 & $\{0,2,4,5\}$ & 39503.7 & 214.89 & 21058.00 & 31 & 139.46 & 463.79 & 450.25 \\
\hline \multirow[t]{6}{*}{0.75} & 0 & $\{4,6,7,8\}$ & 80276.6 & 55.44 & 705.55 & 16 & 77.67 & 231.97 & 15.1 \\
\hline & 0.2 & $\{-,-,-,-\}$ & $71483.3^{5.44 \%}$ & 78.10 & - & 49 & 9.72 & 745.34 & - \\
\hline & 0.4 & $\{-,-,-,-\}$ & $57764.2^{9.65 \%}$ & 118.99 & - & 81 & 5.69 & 1261.38 & - \\
\hline & 0.6 & $\{2,6,7,9\}$ & 48335.9 & 138.12 & 14781.78 & 57 & 7.48 & 877.73 & 12895.37 \\
\hline & 0.8 & $\{0,2,4,5\}$ & 40631.5 & 178.69 & 14652.45 & 59 & 6.61 & 916.28 & 8462.71 \\
\hline & 1 & $\{0,2,4,5\}$ & 43035.9 & 245.25 & 17025.25 & 20 & 117.04 & 293.35 & 541.17 \\
\hline \multirow[t]{6}{*}{0.9} & 0 & $\{4,6,7,8\}$ & 80716.5 & 68.12 & 798.12 & 23 & 73.20 & 339.36 & 50.63 \\
\hline & 0.2 & $\{-,-,-,-\}$ & $69136.1^{60.01 \%}$ & 112.12 & - & 89 & 1.11 & 1411.34 & 13582.25 \\
\hline & 0.4 & $\{-,-,-,-\}$ & $56560.7^{21.21 \%}$ & 105.45 & - & 81 & 1.17 & 1240.82 & 13698.25 \\
\hline & 0.6 & $\{2,4,6,9\}$ & 45798.6 & 128.59 & 14958.25 & 67 & 2.85 & 1064.06 & 14057.85 \\
\hline & 0.8 & $\{0,2,4,5\}$ & 40844.3 & 189.26 & 14899.14 & 61 & 0.21 & 991.95 & 14732.95 \\
\hline & 1 & $\{0,2,4,5\}$ & 51055.4 & 245.42 & 19114.25 & 18 & 83.17 & 266.07 & 5489.24 \\
\hline
\end{tabular}

tractable for computational time. In Table 4 the stopping criteria has been met within the time limit. All runs stop before $1.5 \mathrm{hrs}$. As the problem size increases in Table 5 computational times dramatically increase and exceed the time limit. Still, the quality of the solutions found within the time limit is satisfactory. Similar observations as in Table 1-3 can be observed for larger instances.

Since the model in the present scenario is intended to support decisions at a strategic level the running time are quite satisfactory.

\begin{tabular}{llrrrrrr}
\multicolumn{1}{c}{ Table 4: Computational results for larger sized instance with $n=15, q=3, p \in\{3,5\}}$. \\
\hline \multicolumn{1}{c}{$n$} & \multicolumn{3}{c}{$n=15, q=3, p=3$} & \multicolumn{3}{c}{$n=15, q=3, p=5$} \\
\hline 0.6 & 0 & nr.iter & gap $^{L R}$ & $t^{L R}$ (sec.) & nr.iter & gap $^{L R}$ & $t^{L R}$ (sec.) \\
& 0.25 & 50 & 5.58 & 1482.14 & 22 & 54.46 & 4769.63 \\
& 0.5 & 63 & 4.03 & 2131.15 & 61 & 5.12 & 3445.21 \\
& 0.75 & 41 & 9.11 & 2352.80 & 44 & 8.02 & 3325.46 \\
& 1 & 33 & 145.36 & 4804.16 & 36 & 135.82 & 3613.40 \\
\hline 0.75 & 0 & 18 & 57.18 & 4552.04 & 21 & 51.12 & 4510.41 \\
& 0.25 & 56 & 5.14 & 3785.50 & 51 & 6.01 & 2451.09 \\
& 0.5 & 60 & 2.64 & 3105.04 & 58 & 3.63 & 2782.23 \\
& 0.75 & 43 & 8.57 & 3945.95 & 44 & 7.01 & 2945.30 \\
& 1 & 35 & 145.91 & 4389.23 & 37 & 176.12 & 4904.10 \\
\hline 0.9 & 0 & 22 & 75.24 & 3890.56 & 22 & 81.12 & 3926.41 \\
& 0.25 & 51 & 1.88 & 1852.20 & 55 & 6.38 & 2352.85 \\
& 0.5 & 65 & 4.86 & 2140.99 & 58 & 5.19 & 2421.10 \\
& 0.75 & 45 & 10.45 & 2866.23 & 42 & 15.57 & 3551.52 \\
& 1 & 30 & 140.14 & 4182.36 & 34 & 163.40 & 4948.87 \\
\hline
\end{tabular}

\section{Summary, Conclusion and Future work}

In this paper, we proposed a novel mixed integer linear programming mathematical model for the liner shipping network design problem in a competitive environment. The model has $O\left(n^{4}\right)$ variables and constraints, hence being of tractable size. The model extends and generalizes existing models from the literature of hub-and-spoke network 
Table 5: Computational results for larger sized instance with $n=20, q=5, p \in\{5,7\}$.

\begin{tabular}{llrrrrrr}
\hline & \multicolumn{4}{c}{$n=20, q=5, p=5$} & $n=20, q=5, p=7$ & \\
\hline$\alpha$ & $\lambda$ & nr.iter & \multicolumn{1}{c}{ gap $^{L R}$} & $t^{L R}$ (sec.) & nr.iter & gap $^{L R}$ & $t^{L R}$ (sec.) \\
\hline 0.6 & 0 & 25 & 60.15 & LIMIT & 17 & 112.52 & LIMIT \\
& 0.25 & 20 & 6.17 & LIMIT & 25 & 8.45 & LIMIT \\
& 0.5 & 26 & 5.32 & LIMIT & 26 & 9.31 & LIMIT \\
& 0.75 & 31 & 12.11 & LIMIT & 23 & 14.45 & LIMIT \\
& 1 & 35 & 130.24 & LIMIT & 21 & 121.60 & LIMIT \\
\hline 0.75 & 0 & 17 & 85.16 & LIMIT & 18 & 125.71 & LIMIT \\
& 0.25 & 24 & 7.44 & LIMIT & 16 & 8.70 & LIMIT \\
& 0.5 & 25 & 6.30 & LIMIT & 20 & 9.61 & LIMIT \\
& 0.75 & 14 & 14.19 & LIMIT & 17 & 15.33 & LIMIT \\
& 1 & 26 & 143.60 & LIMIT & 21 & 134.41 & LIMIT \\
\hline \multirow{2}{*}{0.9} & 0 & 20 & 83.76 & LIMIT & 15 & 163.21 & LIMIT \\
& 0.25 & 22 & 5.11 & LIMIT & 17 & 8.81 & LIMIT \\
& 0.5 & 19 & 6.55 & LIMIT & 18 & 11.65 & LIMIT \\
& 0.75 & 25 & 12.63 & LIMIT & 13 & 10.99 & LIMIT \\
& 1 & 25 & 139.46 & LIMIT & 20 & 109.06 & LIMIT \\
\hline
\end{tabular}

design in many aspects such as allowing a general cost structure and permitting fl xibility in the hub-level as well as spoke-level configuration (while maintaining connectivity). Flexibility of this model allows extensions to fi ed time services. It also opens a new horizon to competitive network design of liner shipping services as a prototyping tool. The objective function aims at maximizing the customer attractiveness in terms of shorter travel times and cheaper transportation costs. To the best of our knowledge there has not been any similar work in the literature of liner shipping network design which takes into account cost and service time. In order to solve the problem faster we proposed a Lagrangian decomposition method together with a primal bound generation procedure. An important feature of this solution method is to reduce the problem size in the reduction phase by eliminating several variables, hence reducing the decision space and limiting memory consumption significantl. The lower bound heuristic, which is a MIP solved to optimality by CPLEX, generates feasible solutions, and their quality can easily be verified Due to the inherent decomposable structure of the problem and the big-M nature of the model, Lagrangian relaxation has been chosen as an appropriate solution method. The computational results indicate that our method has been very successful in obtaining tight bounds and outperforms standard solvers such as CPLEX in most cases.

In the future, it will be interesting to develop models of gradual hub location problems, joint deployment and competitive network design for liner shipping. We will also develop solution methods based on column generation and cross decomposition. Moreover, meta-heuristic solution methods deserve to be taken into account.

\section{Acknowledgement}

This study is partially supported by the research Grant WBS No. R-264-000-244-720 from the NOL Fellowship Programme. Authors are also thankful of Prof. Anastassios N. Perakis at the department of Engineering for the Marine Environment of University of Michigan for his useful comments. The last author would like to thank The Danish Council for Strategic Research, The Programme Commission on Strategic Growth Technologies, for their support to the ENERPLAN project.

Alumur, S., Kara, B., Karasan, O., 2009. The design of single allocation incomplete hub networks. Transportation Research Part B.

Alumur, S., Kara, B. Y., 2008. Network hub location problems: The state of the art. European Journal of Operational Research 190 (1), 1-21.

Aversa, R., Botter, H., Haralambides, R., Yoshizaki, H., 2005. A mixed integer programming model on the location of a hub port in the east coast of south america. Maritime Economics and Logistics 7, 1-18.

Aykin, T., 1994. Lagrangian relaxation based approaches to capacitated hub-and-spoke network design problem. European Journal of Operational Research 79 (3), 501-523.

Calık, H., Alumur, S. A., Kara, B., Karasan, O., 2008. A tabu-search based heuristic for the hub covering problem over incomplete hub networks. Computers \& Operations Research.

Campbell, J., 2005. Strategic network design for motor carriers. Logistics Systems: Design and Optimization. Springer, Ch. 6.

Campbell, J. F., 2009. Hub location for time definit transportation. Computers and Operations ResearchArticle in Press. 
Choong, S., Cole, M., Kutanoglu, E., 2002. Empty container management for intermodal transportation networks. Transportation Research Part E $38(6), 423-438$

Contreras, I., Díaz, J., Fernández, E., 2008. Lagrangean relaxation for the capacitated hub location problem with single assignment. OR-Spectrum (to appear).

Cunha, C., Silva, M., 2007. A genetic algorithm for the problem of configurin a hub-and-spoke network for a ltl trucking company in brazil. European Journal of Operational Research 179, 747-758.

Drezner, T., Drezner, Z., 2001. A note on applying the gravity rule to the airline hub problem. Journal of Regional Science 41, 67-73.

Eiselt, H., Marianov, V., 2008. A conditional p-hub location problem with attraction functions. Computers \& Operations Research In Press, Corrected Proof, -

Elhedhli, S., Hu, F., 2005. Hub-and-spoke network design with congestion. Computers and Operations Research 32, $1615-1632$.

Fisher, M. L., 1981. The lagrangian relaxation method for solving integer programming problems. Management Science 27 (1), 1-18.

Fisher, M. L., 2004. The lagrangian relaxation method for solving integer programming problems. Management Science 50 (12), $1861-1871$.

Gelareh, S., 2008. Hub location models in public transport planning. Ph.D. thesis, Universitätsbibliothek.

Gelareh, S., Nickel, S., 2007. A benders decomposition for hub location problems arising in public transport. In: Proceeding of GOR2007.

Gelareh, S., Nickel, S., 2008a. Multi-period public transport planning: A model and greedy neighborhood heuristic approaches. Tech. rep., Department of Optimization, Fraunhofer Institute for Industrial Mathematics (ITWM), D 67663 Kaiserslautern, Germany.

Gelareh, S., Nickel, S., 2008b. New approaches to hub location problems in public transport planning. Tech. rep., Department of Optimization, Fraunhofer Institute for Industrial Mathematics (ITWM), D 67663 Kaiserslautern, Germany.

Goldman, A., 1969. Optimal location for centers in a network. Transportation Science 3, 352-360.

Guignard, M., 2003. Lagrangian relaxation. TOP 11(2), 151-228.

Hakimi, S. L., 1964. Optimum locations of switching centers and the absolute centers and medians of a graph. Operations Research 12 , $450-459$.

Hall, R., 1989. Configuratio of an overnight package air network. Transportation Research A 23, 139-149.

Hsu, C. ., Hsieh, Y. ., 2007. Routing, ship size, and sailing frequency decision-making for a maritime hub-and-spoke container network. Mathematical and Computer Modelling 45 (7-8), 899-916.

Imai, A., Nishimura, E., Papadimitriou, S., Liu, M., 2006. The economic viability of container mega-ships. Transportation Research Part E 42 (1), $21-41$.

Imai, A., Shintani, K., Papadimitriou, S., 2009. Multi-port vs. Hub-and-Spoke port calls by containerships. Transportation Research Part E.

Iyer, A., Ratliff, H., 1990. Accumulation point location on tree networks for guaranteed time distribution. Management Science 36(8), 958-969.

Jaillet, P., Song, G., Yu, G., 1996. Airline network design and hub location problems. Location Science 4 (3), $195-212$.

Jeong, S., Lee, C., Bookbinder, J., 2007. The european freight railway system as a hub-and-spoke network. Transportation Research Part A 41 (6), 523-536.

Konings, R., 2006. Hub-and-spoke networks in container-on-barge transport. No. 1963.

Kuby, M., Gray, R., 1993. The hub network design problem with stopovers and feeders: The case of federal express. Transportation Research A 27 (1), 1-12.

Marianov, V., Serra, D., ReVelle, C., 1999. Location of hubs in a competitive environment. European Journal of Operational Research 114 (2), 363-371.

Marín, A., 2005b. Uncapacitated euclidean hub location: Strengthened formulation, new facets and a relax-and-cut algorithm. Journal of Global Optimization 33, 393-422.

Nickel, S., Schobel, A., Sonneborn, T., 2001. Hub location problems in urban traffic networks. Mathematics Methods and Optimization in Transportation Systems. Kluwer Academic Publishers., Ch. 1.

O’Kelly, M., 1986a. The location of interacting hub facilities. Transportation Science 20 (2), 92-105.

O'Kelly, M., 1986b. Activity levels at hub facilities in interacting networks. Geographical Analysis 18 (4), $343-356$.

O'Kelly, M., 1987. A quadratic integer program for the location of interacting hub facilities. European Journal of Operational Research $32,393-404$.

O'Kelly, M., Miller, H., 1991. Solution strategies for the single facility minimax hub location problem. Papers in Regional Science 70(4), 367-380.

Pirkul, H., Schilling, D., 1998. An efficient procedure for designing single allocation hub and spoke systems. Management Science 44(12), 235242.

Powell, W., Sheffi, Y., 1983. The load planning problem of motor carriers: Problem description and a proposed solution approach. Transportation Research A 17, 471-480.

Racunica, I., Wynter, L., 2005. Optimal location of intermodal freight hubs. Transportation Research Part B 39 (5), $453-477$.

Sasaki, M., Fukushima, M., December 2001. Stackelberg hub location problem. Journal of the Operations Research Society of Japan 44 (4), $390-405$.

Sasaki, M., Fukushima, M., 2003. On the hub-and-spoke model with arc capacity constraints. Journal of the Operations Research Society of Japan 46 (4), 409-428

Sasaki, M., Suzuki, A., Drezner, Z., 1999. On the selection of hub airports for the airline hub-and-spoke system. Computers \& OR 26, $1411-1422$.

Takano, K., Arai, M., 2008. A genetic algorithm for the hub-and-spoke problem applied to containerized cargo transport. Journal of Marine Science and Technology.

UNCTAD (Ed.), 2008. Review Of Maritime Transport 2008. United Nations Conference on Trade and Development (UNCTAD) Geneva and New York.

Wagner, B., 2008. A note on location of hubs in a competitive environment. European Journal of Operational Research 184(1), 57-62.

Yaman, H., 2009. The hierarchical hub median problem with single assignment. Transportation Research Part B: Methodological In Press, Corrected Proof, - 
A new mixed integer programming formulation is proposed for hub-and-spoke network design in a competitive environment. It addresses competition between a newcomer liner service provider and an alliance, both operating on hub-and-spoke networks. The newcomer company maximizes its market share - proportional to service time and transportation cost - by locating a predefined number of hubs at candidate ports and designing its network. While general-purpose solvers do not solve instances of even small size, an accelerated lagrangian method coupled with a primal heuristic obtains very good bounds. Our computational experiments on real instances of practical size indicate superiority of our approach.

ISBN 978-87-90855-73-4

DTU Management Engineering

Department of Management Engineering

Technical University of Denmark

Produktionstorvet

Building 424

DK-2800 Kongens Lyngby

Denmark

Tel. +4545254800

Fax +4545933435

www.man.dtu.dk 